\title{
Severe graft-versus-host disease of the lower intestinal tract after pomalidomide administration in a plasma cell leukemia patient following bone marrow transplantation
}

Hiroto Ishii, Hiroki Yokoyama, Atsushi Katsube, Tadahiro Gunji, Takeshi Saito, Shingo Yano

Division of Clinical Oncology and Hematology, Department of Internal Medicine, The Jikei University School of Medicine, Tokyo, Japan

\begin{abstract}
Posttransplant treatment is performed to treat hematopoietic diseases but can lead to allogeneic-specific complications in addition to those seen in a non-transplant setting. Immunomodulatory drugs (IMiDs) activate cytotoxic $T$ cells and suppress regulatory $T$ cells. The optimal timing and optimal dose of IMiDs after allogeneic transplantation (allo-HSCT) to reduce complications and increase antitumor efficacy are difficult to determine because the degree of recovery of donor immune cells varies depending on the time after allo-HSCT.

We experienced a patient with allo-HSCT who developed severe late acute graft-versus-host disease (GVHD) of the lower intestinal tract after receiving pomalidomide as a posttransplant therapy eight months after alloHSCT. It is possible that pomalidomide induced acute GVHD by altering the activity of donor immune cells. This first case report highlights that the use of pomalidomide after allo-HSCT may lead to severe late acute GVHD. When pomalidomide is used after allo-HSCT, it is desirable to start with a small dose and gradually increase the dose while monitoring cytokine and lymphocyte subsets for the onset of GVHD.
\end{abstract}

Key words bone marrow transplantation, plasma cell leukemia, pomalidomide, graft-versus-host disease, posttransplant treatment

Submitted June 6, 2021; Accepted August 3, 2021; Published online November 25, 2021; Issued online November 25, 2021

Correspondence: Hiroto Ishii, Division of Clinical Oncology and Hematology, Department of Internal Medicine, The Jikei University School of Medicine, 3-19-18, Nishi-Shinbashi, Minato-ku Tokyo, 105-8471, Japan, E-mail: h-ishii@jikei.ac.jp

\section{Introduction}

Selected patients with primary plasma cell leukemia (PPCL) who relapse after autologous peripheral blood stem cell transplantation (ASCT) are indicated for allogeneic hematopoietic stem cell transplantation (alloHSCT $)^{1}$. According to reports from the Center for International Bone and Marrow Transplant Research (CIBMTR), the median progression-free survival (PFS) for PPCL after allo-HSCT has been consistently around 12 months in the era of novel agents ${ }^{2,3}$. Because the PFS after allo-HSCT is not satisfactory, administration of new molecular targeting agents such as daratumumab and elotuzumab, and/or immunomodulatory drugs (IMiDs) for post allo-HSCT is a therapeutic challenge. However, there are several issues to clarify regarding posttransplant therapy with novel agents, such as whether there is a concomitant increase in infection or graft-versus-host disease (GVHD), and the appropriate starting time. Therefore, the best therapeutic strategy in this setting remains unclear.

\section{Case Presentation}

A 56-year-old Japanese woman was diagnosed with IgD- $\lambda$ PPCL harboring $\mathrm{t}(11 ; 14)$. Computed tomography revealed no extramedullary disease. After four courses of induction therapy with cyclophosphamide, bortezomib, and dexamethasone, the patient achieved a very good partial response. She received high-dose chemotherapy with melphalan followed by ASCT. Maintenance therapy comprised lenalidomide and low-dose dexamethasone.

After three months of ASCT, abnormal plasma cells reappeared in the marrow and peripheral blood. In addition, extramedullary disease of the fourth cervical verte- 

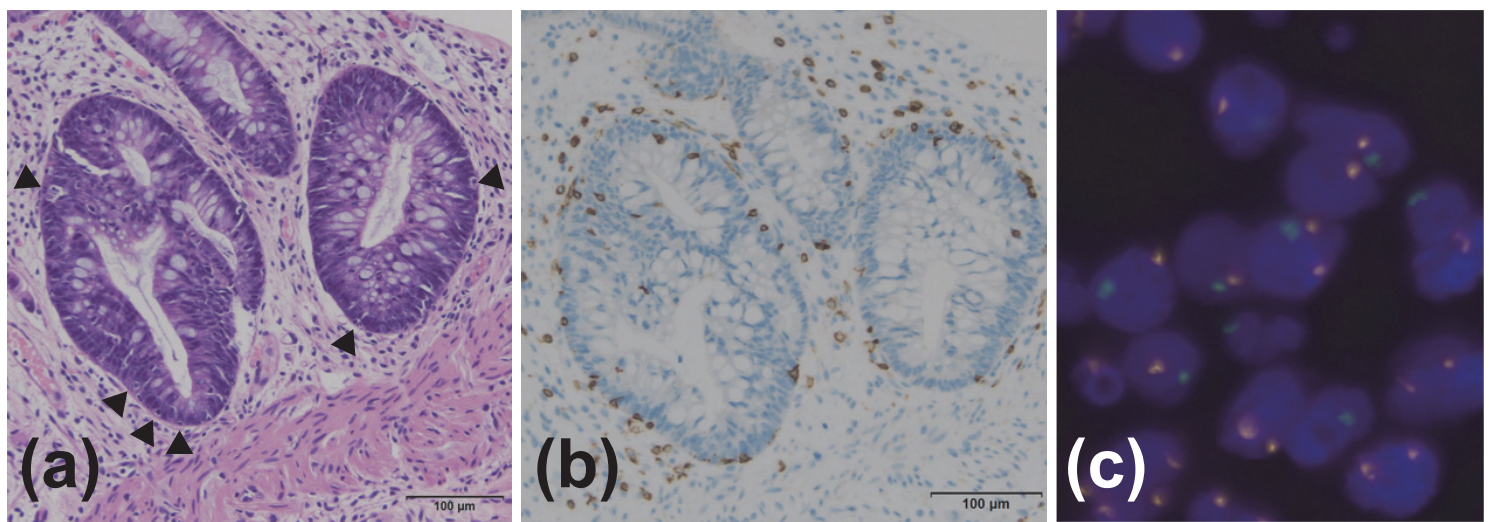

Figure 1.

The histological findings showed epithelial cell apoptosis in a colonic biopsy specimen, edema in the lamina propria, and scattered apoptotic bodies (arrowhead) (hematoxylin-eosin staining). (a)

The histological findings showed CD8-positive lymphocyte infiltration in the crypt (anti-CD8 staining). (b)

$\mathrm{Y}$ chromosome was detected in these lymphocytes (FISH probes for the $\mathrm{Y}$ chromosome, green). (c)

bra occurred. Following salvage treatment with ixazomib, daratumumab, lenalidomide, dexamethasone, cyclophosphamide, etoposide, and cisplatin, a partial response was achieved. She underwent bone marrow transplantation (BMT; total nucleated cells, $4.08 \times 10^{8} /$ $\mathrm{kg}$ ) from an unrelated HLA $8 / 8$ allele-matched man. The conditioning regimen comprised 6 Gy of total body irradiation and $140 \mathrm{mg} / \mathrm{m}^{2}$ melphalan. Prophylaxis against GVHD included tacrolimus and short-term methotrexate. Complete remission occurred on day 76 . Although acute GVHD developed (overall grade II, skin 1 , gut 1, liver 0 ), steroid intervention was unnecessary. GVHD resolved on day 50 after BMT. Because of the high-risk of recurrence, three courses of ELd therapy [Elotuzumab $(10 \mathrm{mg} / \mathrm{kg} /$ day on days $1,8,15$, and 22 , during the first two cycles; then on days 1 and 15 during the third cycle) plus lenalidomide $(10 \mathrm{mg} /$ day on days 1-21) and dexamethasone (20 mg/day on days 1 , 8,15 , and 22)] were started from day 83 after BMT as a consolidation therapy.

She experienced no adverse events such as GVHD recurrence or infection. However, five months after BMT, she relapsed with an intravertebral canal tumor, without marrow or peripheral blood recurrence. Because she demonstrated resistance to salvage chemotherapy (high-dose methotrexate and cytarabine), local radiation therapy (27 Gy) was performed to achieve complete remission. Pd therapy [pomalidomide $(3 \mathrm{mg} /$ day on days 1-21) plus dexamethasone (20 mg/day on days $1,8,15$, and 22)] was started under $0.5 \mathrm{mg} /$ day oral tacrolimus from eight months after BMT. Abdominal pain appeared 16 days after initiating pomalidomide therapy, and diarrhea (up to $4.3 \mathrm{~L}$ /day) began the next day. Pd therapy was therefore discontinued. A colonoscopy performed 26 days after pomalidomide administration revealed mucosal edema, erosion, and shallow ulceration from the sigmoid colon to the rectum. Histopathological examination of several biopsy specimens of the colon revealed negative immunostaining for cytomegalovirus and intense infiltration of lymphocytes and scattered apoptosis of epithelial cells in the lamina propria (Figure 1). Accordingly, grade III GVHD of the lower intestinal tract was diagnosed. First-line treatment of 2 $\mathrm{mg} / \mathrm{kg} /$ day methylprednisolone and tacrolimus was administered, which was changed from oral tablets to continuous intravenous injections 26 days after pomalidomide administration due to the large amount of diarrhea and concern that the blood concentration level of tacrolimus could not be maintained by oral medication. Since there was no improvement for two weeks and her general condition deteriorated, it was necessary to administer infliximab therapy $(5 \mathrm{mg} / \mathrm{kg}$ on the $41 \mathrm{st}$, 56th, and 69th days after pomalidomide administration) in the intensive care unit. Diarrhea gradually reduced after the therapy, and complete remission of GVHD was clinically established on day 34 after initiating infliximab administration (Figure 2). Two months after Pd therapy, her PPCL relapsed as disseminated meningeal lesions, and she died one year after BMT.

\section{Discussion}

Maintenance therapy after allo-HSCT is used not only due to its direct antitumor effect, but also to activate donor cells to enhance the graft-versus tumor effect. GVHD is initiated by T cells sensitized to allogeneic antigens, suppressed by NK cells, and controlled by regulatory $\mathrm{T}$ cells $\mathrm{s}^{4}$. Pomalidomide and elotuzumab can be used together to activate T cells and NK cells in an additive manner, resulting in a higher antitumor effect ${ }^{5}$. It is not completely clear when and how IMiDs after allo-HSCT affect GVHD. 


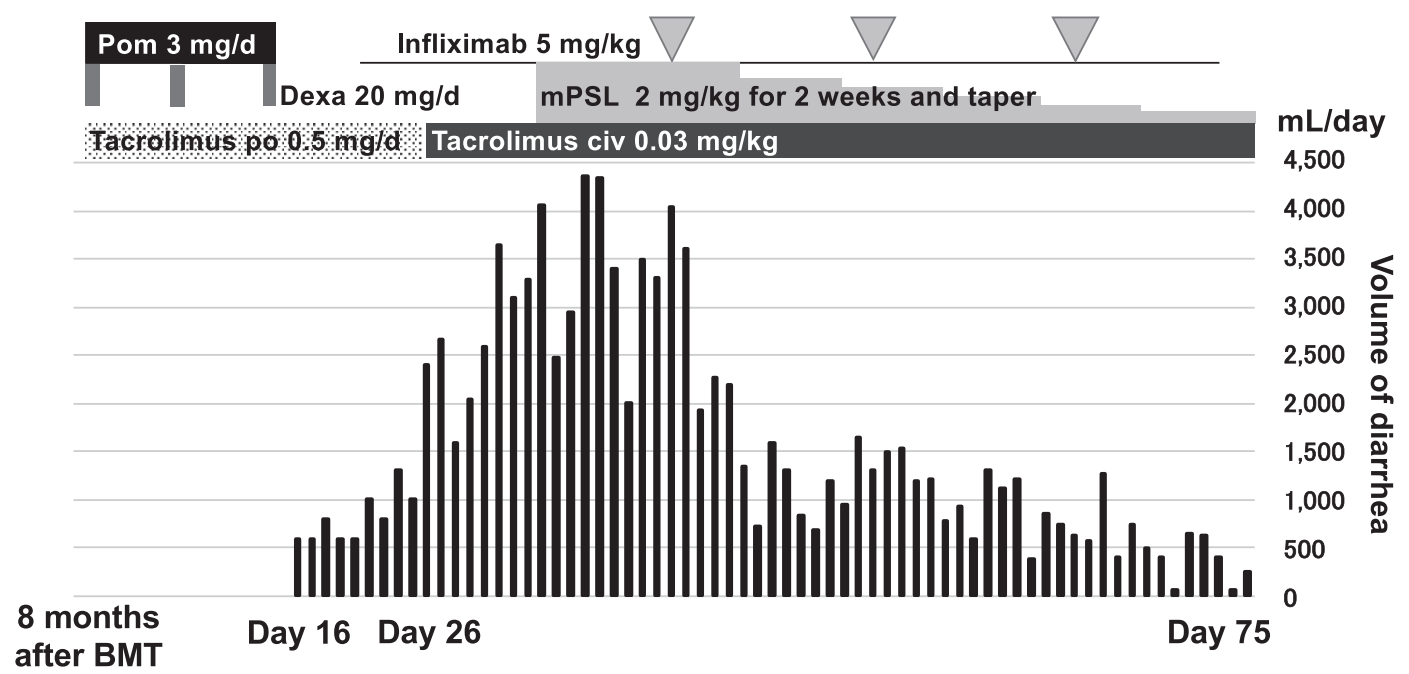

Days after initiating Pd therapy

Figure 2. Clinical course

On the 16th day after initiating Pd therapy, abdominal pain appeared and the volume of diarrhea gradually increased thereafter. After diagnosis of lower intestinal tract GVHD by colonoscopy (day 26 after Pd therapy), tacrolimus and methylprednisolone were administered. However, since there was no improvement, infliximab was additionally administered. GVHD went into complete remission on day 60 after the onset of diarrheal symptoms.

BMT, bone marrow transplantation; civ, continuous intravenous infusion; Dexa, dexamethasone; mPSL, methylprednisolone; Pd, pomalidomide and dexamethasone; Pom, pomalidomide.

Thirty allo-HSCT patients with multiple myeloma in the HOVON 76 trial were administered lenalidomide at $10 \mathrm{mg}$ daily for 21 days in 28-day cycles, and the median time from allo-HSCT to the start of lenalidomide maintenance was three months ${ }^{6}$. In this study, $43 \%$ of patients developed GVHD and needed to discontinue lenalidomide. On the other hand, in a phase I/II dosefinding study, 24 patients with multiple myeloma who had undergone allo-HSCT were treated with lenalidomide starting six months after allo-HSCT, and the maximum tolerable dose was $5 \mathrm{mg}^{7}$. In this study, immune monitoring showed an increase in $\mathrm{CD} 4+$ and $\mathrm{CD}$ $8+\mathrm{T}$ cells within the first week of lenalidomide administration followed by a delayed increase in $\mathrm{T}$ regulatory cells. Other research also revealed that the IMiDs lenalidomide and pomalidomide enhance $\mathrm{T}$ helper type1 cytokine production ${ }^{8}$, increase memory $\mathrm{CD} 4+$ and $\mathrm{CD}$ $8+\mathrm{T}$ cells $\mathrm{s}^{9}$, and decrease $\mathrm{T}$ regulatory cells ${ }^{10}$. These findings suggest that IMiDs may have been involved in the development and refractoriness of GVHD in this patient. There was a transient increase in the absolute peripheral blood lymphocyte counts after Pd therapy (data not shown), and it is conceivable that pomalidomide increased $\mathrm{CD} 4+$ and $\mathrm{CD} 8+\mathrm{T}$ cells, leading to the development of acute GVHD in this case.

The use of lenalidomide after allo-HSCT did not induce GVHD, whereas the use of pomalidomide after allo-HSCT led to severe GVHD. It is difficult to clarify the possible mechanism responsible for this difference because we did not examine the peripheral blood lym- phocyte subsets and cytokines during the clinical course in this case, although the mechanism may have been related to the concomitant use of elotuzumab. Therefore, when using IMiDs after allo-HSCT, it is necessary to pay attention to the onset of GVHD, and it may be important to measure cytokines over time and/or analyze lymphocyte subsets. Although IMiDs may also be a treatment option for chronic GVHD ${ }^{11}$, chronic GVHD differs from acute GVHD in the cells, and the mechanisms involved and onset and severity of GVHD might be different depending on the timing of administration and dose.

To the best of our knowledge, this is the first case report suggesting that the use of pomalidomide after alloHSCT may lead to severe late acute GVHD. When administering pomalidomide after allo-HSCT, it is desirable to start with a small dose and gradually increase while monitoring for the onset of GVHD.

\section{Author Contributions}

HI, HY, and TS. wrote this manuscript. AK, TG, and SY. revised this manuscript. All authors read and approved the final manuscript.

\section{Ethics for Research and Informed Consent}

This case report was approved by the Ethics Committee of The Jikei University School of Medicine for Biomedical Research (\#10848). Written informed con- 
sent was obtained from this patient for publication.

\section{Conflicts of Interest}

The authors declare no conflict of interest. Disclosure forms provided by the authors are available on the website.

\section{References}

1. Fernández de Larrea C, Kyle RA, Durie BG, Ludwig H, Usmani S, Vesole DH, et al. Plasma cell leukemia: consensus statement on diagnostic requirements, response criteria and treatment recommendations by the International Myeloma Working Group. Leukemia. 2013; 27: 780-91.

2. Mahindra A, Kalaycio ME, Vela-Ojeda J, Vesole DH, Zhang MJ, Li P, et al. Hematopoietic cell transplantation for primary plasma cell leukemia: results from the Center for International Blood and Marrow Transplant Research. Leukemia. 2012; 26: 1091-7.

3. Dhakal B, Patel S, Girnius S, Bachegowda L, Fraser R, Davila $\mathrm{O}$, et al. Hematopoietic cell transplantation utilization and outcomes for primary plasma cell leukemia in the current era. Leukemia. 2020; 34: 3338-47.

4. Blazar BR, Murphy WJ, Abedi M. Advances in graft-versushost disease biology and therapy. Nature Reviews Immunology. 2012; 12: 443-58.

5. Dimopoulos MA, Lonial S, White D, Moreau P, Weisel K, San-Miguel J, et al. Elotuzumab, lenalidomide, and dexamethasone in RRMM: final overall survival results from the phase 3 randomized ELOQUENT-2 study. Blood Cancer Journal. 2020; 10: 91.

6. Kneppers E, van der Holt B, Kersten MJ, Zweegman S, Mei- jer E, Huls G, et al. Lenalidomide maintenance after nonmyeloablative allogeneic stem cell transplantation in multiple myeloma is not feasible: results of the HOVON 76 Trial. Blood. 2011; 118: 2413-9.

7. Wolschke C, Stübig T, Hegenbart U, Schönland S, Heinzelmann M, Hildebrandt Y, et al. Postallograft lenalidomide induces strong NK cell-mediated antimyeloma activity and risk for T cell-mediated GvHD: Results from a phase I/II dosefinding study. Exp Hematol. 2013; 41: 134-42.

8. Quach H, Ritchie D, Stewart AK, Neeson P, Harrison S, Smyth MJ, et al. Mechanism of action of immunomodulatory drugs (IMiDS) in multiple myeloma. Leukemia. 2010; 24: 22-32.

9. Schey SA, Fields P, Bartlett JB, Clarke IA, Ashan G, Knight $\mathrm{RD}$, et al. Phase I study of an immunomodulatory thalidomide analog, CC-4047, in relapsed or refractory multiple myeloma. J Clin Oncol. 2004; 22: 3269-76.

10. Galustian C, Klaschka DC, Meyer B, Labarthe M-C, Bartlett JB, Dalgleish AG. Lenalidomide (Revlimids, CC-5013) and Actimidt (CC-4047) inhibit the function and expansion of $\mathrm{T}$ regulatory (Treg) cells in vitro: implications for anti-tumor activity in vivo. AACR Meeting Abstracts. 2006; 2006: 1147.

11. Curtis LM, Ostojic A, Venzon DJ, Holtzman NG, Pirsl F, Kuzmina ZJ, et al. A randomized phase 2 trial of pomalidomide in subjects failing prior therapy for chronic graft-versushost disease. Blood. 2021; 137: 896-907.

https://doi.org/10.31547/bct-2021-006

Copyright (C)2021 Asia-Pacific Blood and Marrow Transplantation Group (APBMT). This is an open access article distributed under CC BY-NC license (https://creativecommon s.org/licenses/by-nc/4.0/). 